\title{
EVALUACIÓN EN PREESCOLAR Y ESTRATEGIAS PEDAGÓGICAS PARA ATENDER SIGNOS DE ALERTA DE NECESIDADES EDUCATIVAS ESPECIALES EN NIÑOS DEL CANTÓN MORONA EN 2019
}

\author{
Milagros de la Caridad Rodríguez Andino \\ Universidad Católica de Cuenca, Profesor Titular Principal \\ Ph.D. en Ciencias de la Educación. Email: mrodrigueza@ucacue.edu.ec \\ https://orcid.org/0000-0001-9046-2089 \\ Lady Julissa Cobeña Santander \\ Universidad Católica de Cuenca. Estudiante del ciclo de titulación \\ Licenciatura en Educación Inicial. Email: ljcobenas95@est.ucacue.edu.ec \\ Manuel Enrique Quinde Saquicaray \\ Universidad Católica de Cuenca. Estudiante del ciclo de titulación \\ Licenciatura en Educación Inicial. Email: mequindes40@est.ucacue.edu.ec \\ Angie Pamela López Flores \\ Universidad Católica de Cuenca. Estudiante del ciclo de titulación \\ Licenciatura en Educación Inicial. Email: aplopezf32@est.ucacue.edu.ec
}

Para citar este artículo puede utilizar el siguiente formato:

Milagros de la Caridad Rodríguez Andino, Lady Julissa Cobeña Santander, Manuel Enrique Quinde Saquicaray y Angie Pamela López Flores: "Evaluación en preescolar y estrategias pedagógicas para atender signos de alerta de necesidades educativas especiales en niños del Cantón Morona en 2019", Revista Atlante: Cuadernos de Educación y Desarrollo (vol 13, № 2 febrero 2021, pp. 126-142). En línea:

https://www.eumed.net/es/revistas/atlante/2021-febrero/necesidades-educativas-ninos

\section{RESUMEN}

La evaluación del aprendizaje es fundamental para la toma de decisiones, sin embargo, se detectan insuficiencias al utilizar los resultados de la misma para proyectar estrategias que faciliten atender las necesidades educativas especiales (NEE) de algunos niños. El objetivo fue analizar las concepciones de los docentes sobre la evaluación del aprendizaje y la aplicación que realizan de las estrategias pedagógicas que permiten atender las NEE en preescolar, para realizar una intervención educativa que favoreciera el desarrollo de destrezas de los infantes en los ámbitos medidos. El estudio fue empírico, con enfoque mixto, se recolectaron datos de dos Centros Infantiles, del Cantón Morona de Ecuador, utilizando encuestas y entrevistas sobre el tema en estudio, aplicadas a 9 docentes y la observación a una muestra de 60 niños para conocer el desarrollo de sus destrezas y a partir de las deficiencias detectadas aplicar una 
intervención educativa que finalizó con una observación denominada postest. Los principales resultados en la investigación fueron: la mayoría de los docentes conocen el proceso de evaluación, pero no lo aplican de forma continua; además no tienen conocimiento suficiente sobre las estrategias pedagógicas que se pueden utilizar para atender a las necesidades de cada infante; con respecto al preexperimento al realizar la intervención con las estrategias pedagógicas diseñadas, provoco una mejora en el desarrollo de las destrezas de los alumnos con alguna NEE. Como conclusión se señala la importancia de capacitar a los docentes sobre las estrategias pedagógicas a utilizar tomando en cuenta las necesidades de cada educando para lograr el aprendizaje.

Palabras clave: evaluación de los aprendizajes; necesidades especiales; estrategias pedagógicas; aprendizaje en preescolar

\section{EVALUATION IN PRESCHOOL AND EDUCATIONAL STRATEGIES TO ATTEND ALERT SIGNS OF SPECIAL EDUCATIONAL NEEDS IN CHILDREN OF THE CANTON MORONA IN 2019}

\section{ABSTRACT}

The assessment of learning is essential for decision-making, however, shortcomings are detected when using the results of the same to project strategies that facilitate meeting the special educational needs (SEN) of some children. The objective was to analyze the teachers' conceptions about the evaluation of learning and the application they carry out of the pedagogical strategies that allow to attend the SEN in preschool, to carry out an educational intervention that favors the development of skills of the infants in the measured areas. The study was empirical, with a mixed approach, data were collected from two Children's Centers, of the Morona Canton of Ecuador, using surveys and interviews on the subject under study, applied to 9 teachers and the observation of a sample of 60 children to know the development of their skills and, based on the deficiencies detected, apply an educational intervention that ended with a so-called post-test observation. The main results of the research were: most teachers know the evaluation process, but do not apply it continuously; Furthermore, they do not have sufficient knowledge about the pedagogical strategies that can be used to meet the needs of each infant; With respect to the pre-experiment, when carrying out the intervention with the designed pedagogical strategies, it caused an improvement in the development of the skills of the students with some SEN. As a conclusion, the importance of training teachers on the pedagogical strategies to be used taking into account the needs of each student to achieve learning is pointed out.

Key words: Evaluation; Developing; Needs; Strategies; Learning.

\section{INTRODUCCIÓN}

La evaluación en educación inicial es de gran importancia, es una actividad continua del proceso educativo que fortalecer y consolida los aprendizajes, así como los logros de los objetivos o propósitos en cualquier ámbito de desarrollo del niño. La misma permite evidenciar 
cuáles son las necesidades prioritarias que deben ser atendidas y responder a esas necesidades logrando así el aprendizaje personalizado en cada infante.

En esta investigación se analizarán las siguientes preguntas: ¿Qué concepciones tienen los docentes sobre la evaluación en el nivel inicial?; ¿Las maestras atienden y evalúan a los niños considerando las necesidades educativas especiales que presenten? ¿Es posible que aplicando estrategias pedagógicas para atender las NEE, se logren mejoras en las destrezas de los niños?

El estudio tuvo el propósito de analizar las concepciones de los docentes sobre la evaluación del aprendizaje en el nivel inicial y la aplicación que realizan de las estrategias pedagógicas que permiten atender las necesidades educativas especiales de los niños, para realizar una intervención educativa con adaptaciones curriculares que favorecieran el desarrollo de destrezas de los infantes en los ámbitos medidos. Un primer antecedente es el trabajo de Cabrera (2015) quien en una investigación que tuvo como objetivo analizar los procesos evaluativos realizados por los docentes de Educación Inicial para los niños de 4 a 5 años de edad encontró que "los resultados de la investigación demuestran que el nivel de conocimiento que tienen los docentes sobre la evaluación se encuentra en un rango medio a bajo" (p. 5), además se establecieron dos posiciones contrapuestas por las educadoras, por un lado, algunas de ellas manifiestan que la evaluación en este nivel educativo debería conformarse únicamente de forma cualitativa, pues lo trascendental es el registrar descriptivamente lo que realizan los niños, de manera que se pueda además de emitir juicios de valor correctos de los logros o avances de sus estudiantes, retroalimentar su labor docente. Por otra parte, otro grupo de educadoras, a pesar de saber que la evaluación en educación inicial debe llevarse de forma cualitativa, consideran que sería mejor que se califique en base a una notación numérica, pues así los padres de familia darían mayor importancia a este nivel educativo, y los niños y niñas podrían demostrar sus conocimientos más fácilmente (Cabrera, 2015).

Un segundo antecedente analizado fue el trabajo de Bonilla (2015) en un estudio que se centra en el diseño de una capacitación organizada para los maestros de Educación Inicial, el propósito fue el de brindarles las herramientas y estrategias necesarias para que puedan dar una mejor respuesta educativa, que favorezca la inclusión de los niños con necesidades educativas especiales (NEE). "Los resultados obtenidos en el diagnóstico revelaron que solo el $8 \%$ de las docentes se encontraban con una preparación aceptable en relación a las temáticas planteadas, evidenciándose en el resto una gran demanda de preparación” (p. 10). Los datos obtenidos sirvieron como base para el diseño de la capacitación. Una vez ejecutada esta se aplicó una evaluación final (postest) que comprendía las mismas preguntas del pretest, esto permitió observar los cambios conceptuales y actitudinales que se generaron en las educadoras a través de la capacitación. Este proyecto intento dar respuesta a la carencia de capacitación que tienen los docentes de educación inicial sobre inclusión educativa brindando herramientas y estrategias que favorezcan la atención de todos los niños con necesidades educativas especiales asociadas y no asociadas a la discapacidad. Se evidencio en las docentes una gran necesidad de información y capacitación en temas inclusivos y NEE. 


\section{Marco teórico conceptual}

En relación al concepto de evaluación, existe una amplia bibliografía que lo valora desde diferentes perspectivas. En esta investigación se plantean algunas conceptualizaciones de evaluación para el nivel de educación preescolar.

Altamirano (2015), así como documentos del Ministerio de Educación de Ecuador (2014) y del Ministerio de Educación del Perú (2006) han hablado del concepto de evaluación del aprendizaje para la educación inicial coincidiendo en que es un proceso en el cual la educadora evalúa los aprendizajes y destrezas que el niño va adquiriendo en el proceso de aprendizaje, donde la evaluación consiste en un proceso sistemático de recogida de datos disponiendo de información continua y significativa valorando respecto a ella y tomando decisiones adecuadas. Además, la evaluación permite conocer que tan eficaz ha sido el trabajo de la docente, de manera que la maestra conozca en qué nivel de aprendizaje se encuentran los niños, con la finalidad de ayudar al infante a alcanzar las destrezas previstas en el currículo.

Para esta investigación se adopta el concepto dado por el MinEduc (2014) donde se afirma que la evaluación:

Se concibe como un juicio de valor que permite conocer qué tan eficiente ha sido la labor docente en la formación de los niños, conocer el nivel de desarrollo y aprendizaje en el que estos se encuentran y brindar información a quienes lo necesiten. Esta concepción se ajusta a un enfoque desde el que evaluar no significa medir ni discriminar, se constituye más bien, en un referente para tomar nuevas alternativas para el mejoramiento del trabajo diario del docente. (p. 55)

\section{Importancia de la evaluación y características}

En este trabajo se asume lo señalado por la Secretaría de Estado de Educación de la República Dominicana (2006) donde se habla de la importancia de la evaluación en el proceso de aprendizaje del niño con el fin de mejorar la calidad del proceso educativo:

En el Nivel Inicial, y desde esta perspectiva procesual en el marco de una estrategia globalizadora, se evalúa para tener referentes e informaciones articuladas que posibiliten una valoración justa y fundamentada acerca del proceso que vivencia cada niño y niña con el contexto de la dinámica socio-educativa. Las informaciones obtenidas, analizadas, reflexionadas y correlacionadas aportan para la toma de decisiones oportunas que contribuyen a mejorar la calidad del proceso educativo, en relación a los propósitos curriculares (p. 2).

Existen características que debe cumplir la evaluación en el nivel de educación preescolar, para que su aplicación sea exitosa en el proceso de enseñanza aprendizaje, garantizando el desarrollo integral del niño. Altamirano (2015) manifiesta que la evaluación debe ser:

- Formativa: siempre está orientada a mejorar la enseñanza y aprendizaje buscando el desarrollo integral del niño, formando parte del proceso educativo. 
- Continua: esta se realiza a lo largo de todo el proceso de enseñanza y aprendizaje, comienza con la evaluación inicial y culmina con la evaluación final, pretendiendo medir e interpretar y no calificar.

- Integral: evalúa todo proceso y desarrollo del niño considerando la evaluación de manera holística, este tipo de evaluación está referida al progreso del niño y las capacidades que va adquiriendo.

- Decisoria: debe tener un juicio valorativo permitiendo mejorar los procesos de enseñanza y aprendizaje conociendo los logros y dificultades.

- Flexible e individualizada: aquí se caracteriza el ritmo y estilo de cada niño en el proceso de enseñanza y aprendizaje donde la docente reflexiona sobre su manera de actuar.

\section{Técnicas e instrumentos de evaluación en Educación Inicial}

Para la evaluación correcta de los aprendizajes el docente debe aplicar determinadas técnicas e instrumentos, los cuales aportarán los datos necesarios para conocer el estado del desarrollo de las destrezas de los niños y poder realizar la toma de decisiones que se requiera en cuanto a las estrategias pedagogías a utilizar para atender las necesidades educativas especiales que presenten sus estudiantes. En la figura 1, se resumen las técnicas e instrumentos para la evaluación, a partir de la revisión bibliográfica del currículo de educación inicial establecido por el Ministerio de Educación del Ecuador, MinEduc (2014):

Figura 1:

Técnicas e instrumentos de evaluación en Educación Inicial

\begin{tabular}{|c|c|}
\hline Técnicas & Instrumentos: \\
\hline $\begin{array}{l}\text { La entrevista: Se realiza a los padres de familia de los } \\
\text { niños/as, al empezar el año escolar; se trata de un diálogo } \\
\text { amable y empático para generar un clima de confianza y } \\
\text { obtener la información necesaria para conocer el contexto } \\
\text { en el que se encuentra el niño/a. }\end{array}$ & $\begin{array}{l}\text { Ficha de entrevista: sirve para conocer al niño/a y a su } \\
\text { familia, asi como la percepción que tiene la familia o adulto } \\
\text { responsable de su niño, las expectativas que tiene del centro } \\
\text { educativo, lo comprometido que se encuentra en el proceso } \\
\text { de enseñanza aprendizaje de su hijo. }\end{array}$ \\
\hline \multirow{3}{*}{$\begin{array}{l}\text { La observación: puede ser libre, espontánea o planificada; } \\
\text { se utiliza para evaluar algún comportamiento especifico, } \\
\text { destreza, habilidad, actitudes. }\end{array}$} & $\begin{array}{l}\text { Anecdotario: describe comportamientos o conductas } \\
\text { importantes del niños/a dentro y fuera del salón relacionado } \\
\text { con actuaciones extraordinarias e incidentes significativos } \\
\text { dentro del proceso de enseñanza aprendizaje. }\end{array}$ \\
\hline & $\begin{array}{l}\text { Lista de cotejo: Sirve para registrar la presencia o ausencia } \\
\text { de actitudes, caracteristicas y destrezas puntuales en } \\
\text { relación a los tres ejes de desarrollo y aprendizaje; puede ser } \\
\text { usada al inicio como diagnóstico y durante el proceso. }\end{array}$ \\
\hline & $\begin{array}{l}\text { Escala de estimación o tabla cualitativa de destrezas: } \\
\text { consiste en un cuadro individual donde se registran las } \\
\text { destrezas que se desea alcanzar y la escala de valor } \\
\text { cualitativo que indica en qué parte del proceso se } \\
\text { encuentran los niños. }\end{array}$ \\
\hline
\end{tabular}

Portafolio: consiste en una carpeta con la selección planificada de los trabajos que ha realizado cada niño/a durante el periodo educativo; esta muestra evidencia el proceso de los aprendizajes y destrezas adquiridas por los niños/as.

Fuente: Ministerio de Educación del Ecuador (2014)

\section{Necesidades Educativas Especial, su conceptualización}

Cisneros (2017), Villacis (2018) y Luque (2009) han analizado el concepto de necesidades educativas especiales en el cual han coincidido que las necesidades educativas especiales (NEE) son problemas y dificultades que se presentan en un niño y que pueden ser 
transitorias o permanentes. El docente tiene que identificar las dificultades que presenta cada alumno para de esta forma analizar cómo puede ayudarlo, considerando una adaptación curricular planificada de manera que dé respuesta a las NEE de cada infante, facilitando el desarrollo personal en el proceso de enseñanza aprendizaje precisando determinadas ayudas pedagógicas que se requieran.

Para esta investigación se asume el concepto dado por los autores Lopez y Venezuela (2015) quienes expresan:

Se considera que un estudiante presenta NEE cuando por "una amplia variedad de razones" muestra dificultades mayores en comparación al resto de sus compañeros para acceder a los aprendizajes que le corresponden de acuerdo a su edad y requiere para compensar dichas dificultades, apoyos extraordinarios y especializados, que de no proporcionárseles limitan sus oportunidades de aprendizaje y desarrollo. Por lo tanto, las NEE cubren un rango de necesidades que incluyen discapacidades físicas, sensoriales, mentales y cognitivas, así como dificultades del aprendizaje, emocionales y sociales. (p. 43)

\section{Necesidades educativas especiales no asociadas a una discapacidad y asociadas a una discapacidad y las estrategias pedagógicas para la evaluación de los niños que las presentan.}

Con la revisión bibliográfica del trabajo de Morre (2006), de los documentos del MinEduc (2013) sobre las adaptaciones curriculares para estudiantes con necesidades educativas especiales y del documento del Ministerio de Educación del Ecuador (2011) que menciona las estrategias pedagógicas para atender las NEE, se construyó la figura 2, donde se resumen las NEE, como evaluar a los niños que las presentan y las estrategias pedagógicas para atenderlos:

Figura 2

Clasificación de las necesidades educativas especiales 


\begin{tabular}{|c|c|c|c|}
\hline \multicolumn{4}{|c|}{ NEE NO ASOCIADAS A UNA DISCAPACIDAD } \\
\hline & Principales incidencias & Estrategias pedagógicas para la evaluación & Estrategias pedagógicas \\
\hline $\begin{array}{l}\text { Trastorno por } \\
\text { déficit de } \\
\text { atención sin } \\
\text { hiperactividad }\end{array}$ & $\begin{array}{l}\text { - Tiene dificultad para } \\
\text { mantener la atención } \\
\text { durante } 15 \text { min } \\
\text { - Presenta dificultades } \\
\text { para finalizar tareas } \\
\text { - Le cuesta seguir } \\
\text { órdenes e e } \\
\text { instrucciones. } \\
\text { - Suele perder u olvidar } \\
\text { objetos. } \\
\text { Se distrae con } \\
\text { facilidad. }\end{array}$ & $\begin{array}{l}\text { - Asistir al estudiante al momento de la } \\
\text { evaluación para cerciorarse que } \\
\text { comprende las instrucciones. } \\
\text { - Aplicar la evaluación de manera individual } \\
\text { si es necesario. } \\
\text { - Observarlo durante la evaluación para } \\
\text { brindarle apoyo oportuno. } \\
\text { - La evaluación debe estar de acuerdo a las } \\
\text { adaptaciones realizadas para el estudiante }\end{array}$ & $\begin{array}{l}\text { - Ubicarlo lejos de estímulos que le } \\
\text { distraigan } \\
\text { - Evitar llamarle la atención en } \\
\text { público. } \\
\text { - Iniciar la clase con actividades } \\
\text { que favorezcan la atención. } \\
\text { - Dar órdenes claras y precisas } \\
\text { cuyo grado de dificultad esté } \\
\text { acorde a la edad. } \\
\text { - Utilizar diferentes vías de acceso } \\
\text { al aprendizaje (visual, auditivo, } \\
\text { musical, kinestésico, entre otras). }\end{array}$ \\
\hline & $\begin{array}{l}\text { - Altamente creativos e } \\
\text { ingeniosos. } \\
\text { Poca tolerancia frente } \\
\text { al ritmo de aprendizaje } \\
\text { del otro. } \\
\text { Muestran buen } \\
\text { desarrollo de la } \\
\text { memoria. } \\
\text { - Gran imaginación y } \\
\text { fantasía. }\end{array}$ & $\begin{array}{l}\text { - Permitir que formule respuestas que } \\
\text { surjan de su iniciativa y creatividad. } \\
\text { - Hacerle partícipe de su propia evaluación; } \\
\text { los errores lo retroalimentarán } \\
\text { positivamente. } \\
\text { - Promover la autoevaluación }\end{array}$ & $\begin{array}{l}\text { - Aprovechar las salidas } \\
\text { pedagógicas como espacios de } \\
\text { enriquecimiento y búsqueda de } \\
\text { nuevos conocimientos. } \\
\text { - Desarrollar actitudes de respeto } \\
\text { hacia los demás. } \\
\text { - Desarrollar la inteligencia lógico- } \\
\text { matemática a través de } \\
\text { experimentos. }\end{array}$ \\
\hline $\begin{array}{l}\text { Trastorno de } \\
\text { comportamiento }\end{array}$ & $\begin{array}{l}\text { - Desafio a la autoridad. } \\
\text { Molesta } \\
\text { deliberadamente a } \\
\text { otras personas. } \\
\text { - Acusa a otros de sus } \\
\text { emores y } \\
\text { comportamientos. } \\
\text { Tendencia a rabietas } \\
\text { frecuentes. }\end{array}$ & $\begin{array}{l}\text { - Estar pendiente durante toda la evaluación, } \\
\text { puesto que puede empezar a llenar por } \\
\text { llenar. } \\
\text { - Detener la evaluación si le genera mucha } \\
\text { ansiedad, tranquilizarlo y continuar luego } \\
\text { con ella. } \\
\text { - Evaluarlo de manera individual si fuese } \\
\text { necesario. } \\
\text { - Tener en el pupitre solo lo necesario para } \\
\text { la evaluación. }\end{array}$ & $\begin{array}{l}\text { - Ubicarlo en un puesto cercano al } \\
\text { docente y junto a companeros que } \\
\text { influyan en él positivamente. } \\
\text { - Trabajar en espera de tumos. } \\
\text { - Promover espacios de diálogo y } \\
\text { reflexión ante comportamientos } \\
\text { inadecuados, esto mejorará su } \\
\text { autocontrol. } \\
\text { - Evitar ceder o ignorar ante un } \\
\text { comportamiento inadecuado. }\end{array}$ \\
\hline
\end{tabular}

\begin{tabular}{|c|c|c|c|}
\hline & Principales incidencias & Estrategias pedagógicas para la evaluación & Estrategias pedagógicas \\
\hline $\begin{array}{l}\text { Discapacidad } \\
\text { intelectual }\end{array}$ & $\begin{array}{l}\text { - Disminución de sus } \\
\text { habilidades cognitivas. } \\
\text { - Retraso en el } \\
\text { desarrollo en las áreas } \\
\text { de autonomía e } \\
\text { independencia. } \\
\text { - Dificultad en los } \\
\text { procesos de atención y } \\
\text { concentración. } \\
\text { - Ritmo de aprendizaje } \\
\text { más lento. } \\
\text { - Presenta dificultades } \\
\text { en la comprensión de } \\
\text { instrucciones y } \\
\text { órdenes. }\end{array}$ & $\begin{array}{l}\text { - Dar un tiempo mayor para su ejecución. } \\
\text { - Asegurarse que las instrucciones estén } \\
\text { bien comprendidas. } \\
\text { - Adecuar la forma de valorar en función de } \\
\text { las potencialidades. } \\
\text { - Evaluación permanente, donde se priorice } \\
\text { la observación. }\end{array}$ & $\begin{array}{l}\text { - Estimular todos los logros del } \\
\text { estudiante, por pequeños que sean } \\
\text { - Apoyarse en el uso de material } \\
\text { concreto } \\
\text { - Planificar tiempos cortos de } \\
\text { trabajo e ir incrementándolo poco } \\
\text { a poco. } \\
\text { - Respete el ritmo y estilo de } \\
\text { aprendizaje. }\end{array}$ \\
\hline
\end{tabular}




\begin{tabular}{|c|c|c|c|}
\hline $\begin{array}{l}\text { Discapacidad } \\
\text { visual }\end{array}$ & $\begin{array}{l}\text { - Falta de comprensión } \\
\text { de ciertos adverbios } \\
\text { (arriba, abajo, dentro, } \\
\text { fuera, entre otros). } \\
\text { - Mayor habilidad para } \\
\text { percibir a través de los } \\
\text { sentidos del tacto y el } \\
\text { oido. } \\
\text { - Dificultad en el } \\
\text { desplazamiento. }\end{array}$ & $\begin{array}{l}\text { - Priorizar evaluaciones orales sobre las } \\
\text { escritas. } \\
\text { - Permitir tiempo extra. } \\
\text { - Orientar con la lectura de las indicaciones. } \\
\text { - Promover evaluaciones con recursos } \\
\text { informáticos a través de textos hablados y } \\
\text { grabadoras. } \\
\text { - Brindar el apoyo y ayuda solicitada por el } \\
\text { niño, niña y/o adolescente. }\end{array}$ & $\begin{array}{l}\text { - Ampliar los caracteres gráficos } \\
\text { impresos: números, dibujos, en } \\
\text { casos de baja visión. } \\
\text { - Utilizar palabras de guía como } \\
\text { derecha, detrás, arriba para } \\
\text { indicar los adverbios de lugar } \\
\text { (aquí, alli, ahí). } \\
\text { - Ubicarlo en lugares donde no } \\
\text { existan reflejos solares ni de } \\
\text { iluminación que los deslumbren. }\end{array}$ \\
\hline $\begin{array}{c}\text { Deficiencia } \\
\text { motriz }\end{array}$ & $\begin{array}{ll}\text { - } & \begin{array}{l}\text { Movimientos } \\
\text { incontrolados, }\end{array} \\
\text { - } & \text { Dificultades de } \\
\text { coordinación, } & \\
\text { - } & \text { Alcance limitado, } \\
\text { fuerza reducida, } & \\
\text { - } & \begin{array}{l}\text { Dificultad con la } \\
\text { motricidad fina } \\
\text { gruesa. }\end{array}\end{array}$ & $\begin{array}{l}\text { - Criterios de evaluación individualizada. } \\
\text { - Priorizar lo oral sobre lo escrito } \\
\text { dependiendo del tipo de afectación. } \\
\text { - Dosificar el tiempo de evaluación. } \\
\text { - Dar la guía o ayuda requerida solicitada } \\
\text { por el niño, niña y/o adolescente. }\end{array}$ & $\begin{array}{l}\text { - Ubicar al niño en una silla y mesa } \\
\text { apropiadas y cómodas, para lograr } \\
\text { una postura correcta. } \\
\text { - Favorecer el desarrollo de } \\
\text { aprendizajes vivenciales y } \\
\text { significativos. } \\
\text { - Entregarle material de tamaño grande } \\
\text { y color llamativo, textura variada, } \\
\text { resistencia al agarre y grosor } \\
\text { suficiente. }\end{array}$ \\
\hline \multirow{4}{*}{$\begin{array}{l}\text { Trastorno } \\
\text { general del } \\
\text { desarrollo }\end{array}$} & Características & Estrategias pedagógicas para la evaluación & Estrategias pedagógicas \\
\hline & $\begin{array}{l}\text { - Parece no escuchar } \\
\text { cuando se le habla. } \\
\text { - Deja tareas } \\
\text { inconclusas cuando no } \\
\text { son de su agrado. } \\
\text { - Demuestra dificultad } \\
\text { en el desarrollo motor } \\
\text { fino. } \\
\text { Cambia } \\
\text { repentinamente su } \\
\text { estado de ánimo }\end{array}$ & $\begin{array}{l}\text { - Apoyarlo durante la evaluación para } \\
\text { brindarle las ayudas requeridas. } \\
\text { - Asegurarse que las instrucciones sean } \\
\text { comprendidas. } \\
\text { - Adaptar las evaluaciones de acuerdo a sus } \\
\text { necesidades y características. }\end{array}$ & $\begin{array}{l}\text { - Disminuir los ruidos excesivos } \\
\text { - Utilizar material gráfico que le } \\
\text { permitirá hacer asociaciones a } \\
\text { nivel mental. } \\
\text { - Evitar los comportamientos de } \\
\text { sobreprotección. }\end{array}$ \\
\hline & $\begin{array}{l}\text { AUTISMO: } \\
\text { - Dificultad para } \\
\text { comprender } \\
\text { emociones. } \\
\text { - El lenguaje no tiene } \\
\text { función comunicativa. } \\
\text { - Los patrones no } \\
\text { verbales no son } \\
\text { comprendidos. } \\
\text { - Les cuesta desarrollar } \\
\text { habilidades sociales. } \\
\text { Dificultades en los } \\
\text { momentos de } \\
\text { anticipación. }\end{array}$ & $\begin{array}{l}\text { - Adaptar el tiempo en las pruebas o } \\
\text { evaluaciones. } \\
\text { - Dar un ejemplo inicial de lo que se le pide } \\
\text { para evaluar. } \\
\text { - Pedir respuestas no verbales como señalar, } \\
\text { encerrar, construir. } \\
\text { - Enseñar al estudiante diversas formas para } \\
\text { responder a diferentes tipos de pruebas, } \\
\text { evitar exponerlo a modelos de evaluación } \\
\text { que no haya manejado antes. }\end{array}$ & $\begin{array}{l}\text { - Utilizar preferiblemente material } \\
\text { gráfico para ilustrar los conceptos } \\
\text { o situaciones: láminas, videos, } \\
\text { modelos, dibujos, entre otros. } \\
\text { - Utilizar un cronograma de } \\
\text { actividades en forma permanente } \\
\text { para que el estudiante pueda saber } \\
\text { de forma anticipada las rutinas y } \\
\text { actividades que va a realizar. } \\
\text { - Utilizar un lenguaje claro y } \\
\text { concreto para dar órdenes. } \\
\text { Tratar de ser flexibles en el } \\
\text { manejo del tiempo puesto que si } \\
\text { se les presiona, la ansiedad y la } \\
\text { inseguridad se les acentía. }\end{array}$ \\
\hline & \begin{tabular}{|l}
\multicolumn{2}{|c|}{ SÍNDROME } \\
DE DOWN \\
- Requiere reforzar \\
constantemente los \\
aprendizajes para \\
consolidarlos. \\
- Dificultades en la \\
percepción auditiva. \\
- Períodos cortos de \\
atención.
\end{tabular} & $\begin{array}{l}\text { - Otorgar tiempos extras hasta que termine } \\
\text { la evaluación. } \\
\text { - Garantizar la comprensión de las } \\
\text { instrucciones. } \\
\text { - Priorizar los resultados cualitativos. } \\
\text { - Utilizar la música y el arte como } \\
\text { instrumentos de evaluación a través de } \\
\text { representaciones en las que estén } \\
\text { manifiestos los contenidos curriculares. }\end{array}$ & 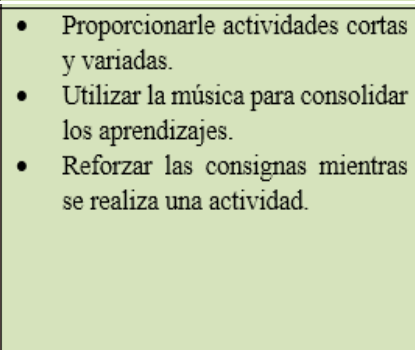 \\
\hline
\end{tabular}

Fuente: (Morre, 2006); MinEduc, (2013); (Ministerio de Educación de Ecuador, 2011) 
Cuando el docente observa que un niño presenta determinadas dificultades de manera continua, durante varios meses, es importante que, si ya ha agotado todos los recursos pedagógicos, didácticos y humanos que estén a su alcance, remita al estudiante a una evaluación psicopedagógica integral.

En el documento elaborado por el Ministerio de Educación de Ecuador (2011) se señala que cuando se observe en los estudiantes lo siguiente: presenta dificultad para aprender; no sigue el ritmo de aprendizaje de sus compañeros de aula; evita relacionarse con sus pares y/o adultos y no participa o presenta un comportamiento agresivo, disociador y otros que interfieren en la dinámica del aula; muestra problemas de salud constantes que afecta su desempeño escolar y cualquier otra manifestación que influye en su proceso de enseñanza aprendizaje se debe remitir a un psicólogo toda la información relevante en un informe con vistas a que el niño sea evaluado, diagnosticado y se puedan establecer las estrategias pedagógicas para la atención del estudiante en base a la NEE que presenta.

\section{METODOLOGÍA}

El estudio siguió un enfoque cuantitativo - cualitativo, de tipo descriptivo -explicativo, con investigación documental y de campo, ya que los datos se recolectaron directamente del lugar donde suceden los hechos y documental porque se trabajó también con la revisión bibliográfica. Se utilizó un diseño de tipo preexperimental, grupo único pretest y postest. La investigación se llevó a cabo en el período de prácticas preprofesionales de los estudiantes de la carrera de Educación Inicial de la Universidad Católica de Cuenca, que participaron en el estudio, en el año 2019.

Se realizaron tres etapas; diagnostico, intervención y validación. Los métodos empíricos utilizados fueron: la experimentación, la encuesta y la entrevista para determinar los problemas que se presentan en los Centros de Educación Infantil (CEI), en relación con el aprendizaje de los niños y conocer las técnicas e instrumentos de evaluación que se aplican, y por último la ficha de observación para medir las destrezas asociadas a los ámbitos de desarrollo y aprendizaje y detectar las NEE de los niños/as. Para esta investigación la población total fue de 134 niños y 9 docentes del nivel inicial 1 de los dos Centros de Educación Inicial de la ciudad de Macas que participaron en el estudio.

Para el procesamiento de los datos cuantitativos se utilizó el Excel para realizar las tablas de distribución de frecuencia y los gráficos.

\section{RESULTADOS}

\section{1ra etapa: Diagnostico (Pretest)}

Se aplicó una encuesta para analizar si los docentes reconocen las NEE y toman en cuenta en las planificaciones a ejecutar en el aula las necesidades de cada niño, el resultado arrojó que: un $11 \%$ manifiesta que existen niños con discapacidad visual y un $89 \%$ considera que no existen niños con NEE, por lo que se evidencia que solo se toman en cuenta las NEE asociadas a una discapacidad, no reconociendo las no asociadas a la discapacidad. En la planificación solo el $67 \%$ de los docentes toma en cuenta las NEE mientras que un $11 \%$ casi 
nunca lo toma en cuenta. Uno de los factores que puede estar asociados a esta situación es la preparación de los docentes en este tema ya que una parte de los docentes manifiesta que solo a veces reciben capacitaciones relacionadas al abordaje e intervención con niños que presentan NEE.

\section{Ficha de observación de los niños según las NEE}

Además, se aplicó una ficha de observación a los niños/as para determinar las NEE que se estaban presentando, se trabajó en las siguientes dimensiones con signos de alerta que debe ser evaluados por un profesional, y el resultado se muestra en la tabla 1:

Tabla 1:

Signos de alerta de las posibles NEE que presentan los niños/as de los centros objetos del diagnóstico

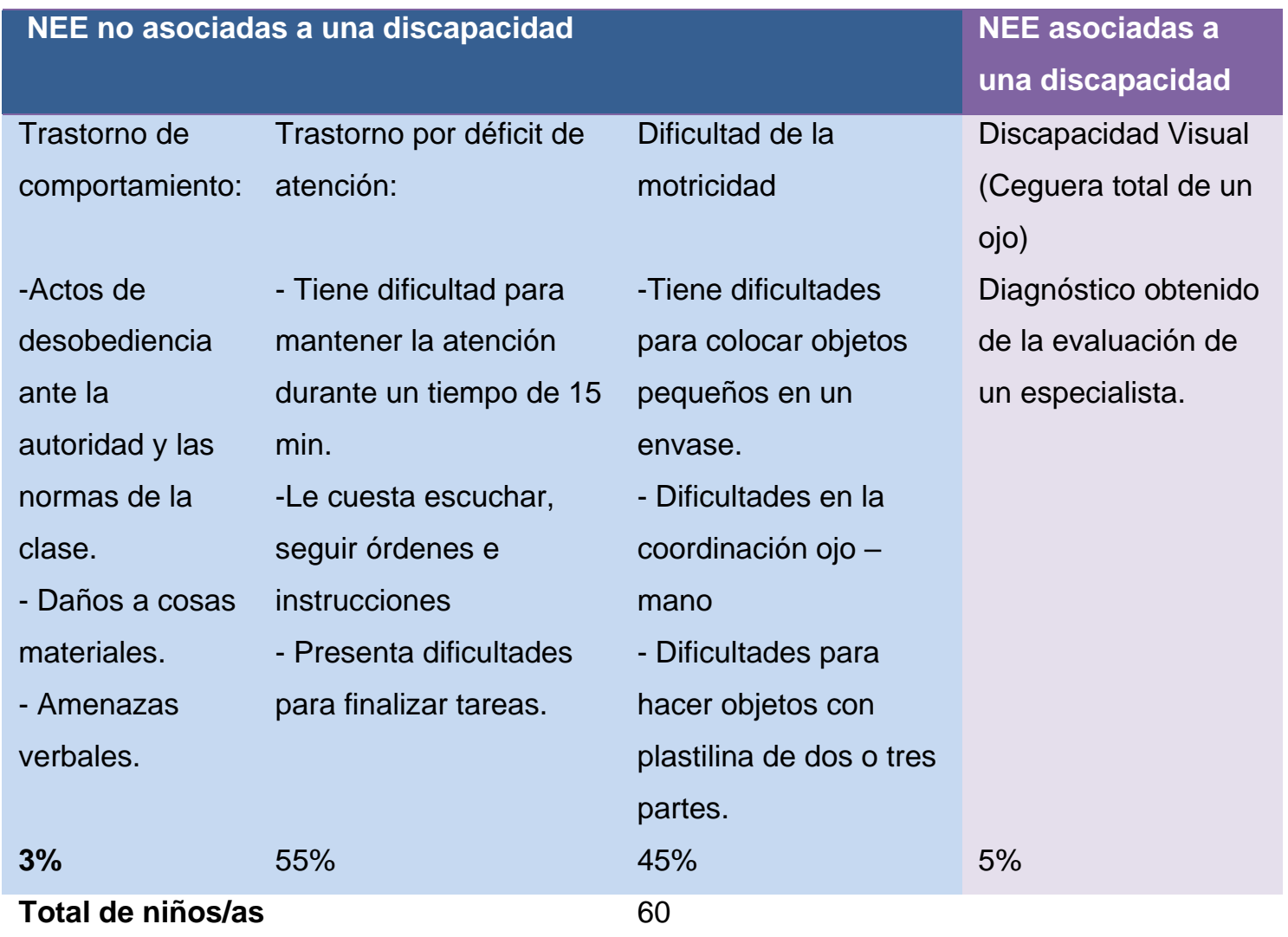

Fuente: Ficha de observación aplicada a los niños de CEI objeto del diagnóstico

Es importante tomar en cuenta que a pesar de que el tiempo que se tuvo para diagnosticar dichas NEE, no fue muy extenso, se observó que ciertos niños tenían signos de alerta que debían ser evaluados por un profesional, donde se pudo presumir que el $3 \%$ de los niños/as presentan trastornos de comportamiento, $55 \%$ presenta trastornos por déficit de atención, el $45 \%$ presenta dificultades de la motricidad fina, y el $5 \%$ presenta discapacidad visual, por lo que se sugirió acceder a un equipo multidisciplinario que brinde un diagnóstico efectivo para determinar y saber qué NEE poseen los niños que presentaron esos signos de alerta. 


\section{Ficha de observación al docente sobre las evaluaciones}

Se aplicó una ficha de observación a los 9 docentes para ver cómo se trabaja con la evaluación tomando en cuenta cuatro indicadores: "la docente utiliza la evaluación de proceso permitiendo obtener información sobre los avances de cada niño"; la docente utiliza la evaluación de proceso permitiendo obtener información sobre los logros alcanzados por cada niño en las destrezas"; "el docente evalúa a cada niño de forma cualitativa para conocer en qué parte del proceso se encuentra" y "al momento de evaluar la docente lo realiza de forma natural tanto en grupo como individual". Los resultados se llevaron a la escala de Likert de frecuencia (siempre (5), casi siempre (4), a veces (3), casi nunca (2), nunca (1)), en la figura 3 , se muestran los resultados.

Figura 3:

Evaluación en escala de Likert de los resultado asociado a la aplicación de los instrumentos de evaluación

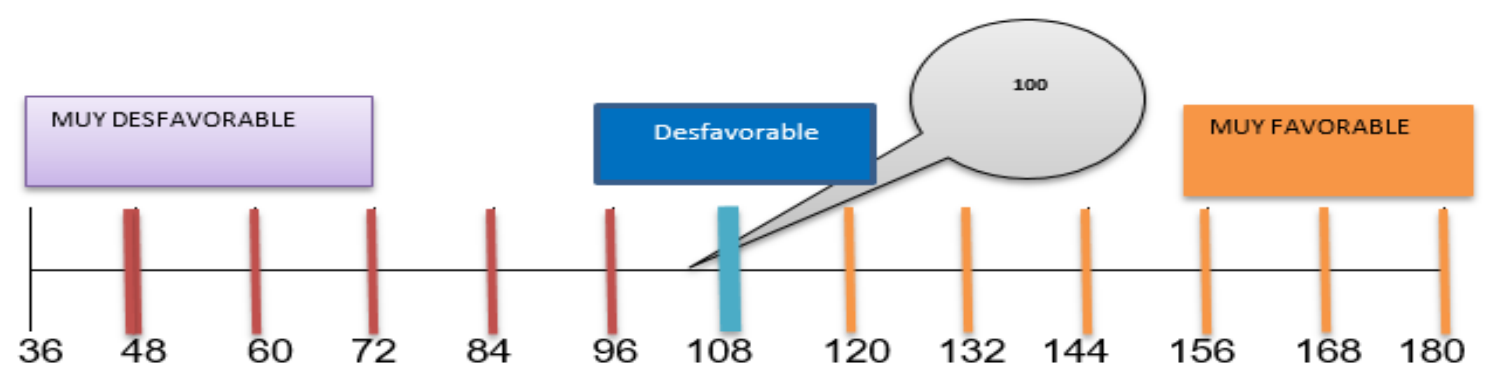

Se puede apreciar que el resultado asociado a la aplicación de los instrumentos de evaluación se ubica en el aspecto desfavorable, la causa pudo estar dada porque las docentes no aplican los instrumentos de evaluación de forma continua y permanente, mediante los cuales pueden verificar o registrar los logros adquiridos por los niños. Esto provoca que las educadoras no tengan una apreciación confiable de los resultados de enseñanza y por tal razón no pueden tomar decisiones curriculares para mejorar el proceso de enseñanzaaprendizaje.

\section{Ficha de observación al docente sobre el tratamiento a las NEE}

Se aplicó una ficha de observación a las 9 docente para ver el tratamiento de las NEE en el aula tomando en cuenta cuatro indicadores: "El docente ejecuta estrategias pedagógicas para optimizar los aprendizajes favoreciendo a los niños con NEE"; "El docente ejecuta estrategias pedagógicas para fortalecer el aprendizaje en grupo"; "El docente hace uso de los instrumentos de la evaluación para conocer el progreso de aprendizaje de los niños con NEE" y "al momento de evaluar el docente lo realiza de forma individual", los resultados se llevaron a la escala de Likert (siempre (5), casi siempre (4), a veces (3), casi nunca (2), nunca (1)), en la figura 4 , se muestran los resultados. 
Figura 4

Evaluación en escala de Likert de los resultados asociado a la atención a las NEE en el aula

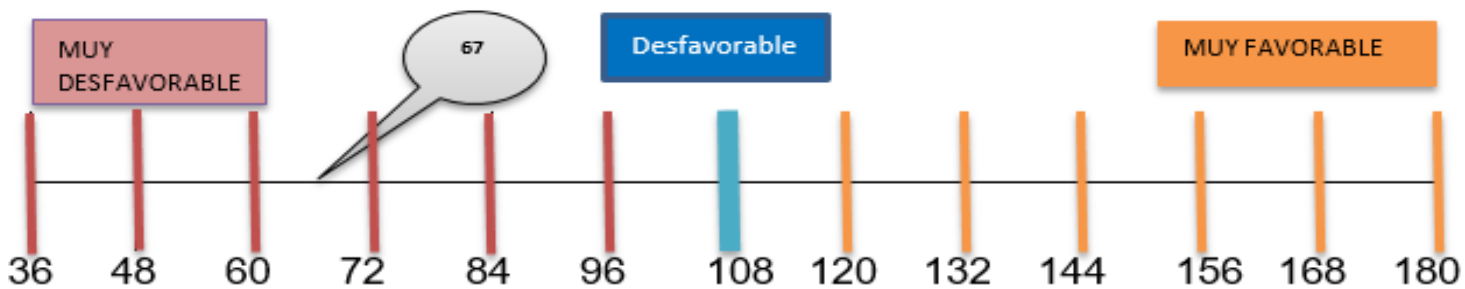

Se observa que los resultados son muy desfavorables debido a que las docentes no aplican todos los indicadores de manera integral para atender y evaluar las NEE. Se deduce por los resultados presentados en la figura 4 que la causa es que las maestras no aplican la evaluación de manera continua y permanente por lo que no pueden detectar ni los logros, ni muchos menos las necesidades educativas que tienen los niños, lo que impide a las educadoras buscar las estrategias y actividades que estén acorde a las necesidades respetando tanto el ritmo como el estilo de aprendizaje para lograr el desarrollo integral de cada infante.

\section{Ficha de observación a los niños sobre el desarrollo de destrezas en los Ámbitos de aprendizaje}

Se aplicó una ficha de observación a los 60 niños de la muestra para medir el desarrollo de las destrezas en los ámbitos de relaciones con el medio natural y en el ámbito compresión y expresión del lenguaje, utilizando una escala de estimación con las siguientes opciones: en Inicio, en Proceso y Adquirido. La tabla 2, presenta los resultados de la primera observación que se denominó Pretest.

Tabla 2:

Resultado de la observación de las destrezas de los niños en los dos Centros

$\begin{array}{llll}\text { Pretest } & \text { I } & \text { P } & \text { A }\end{array}$

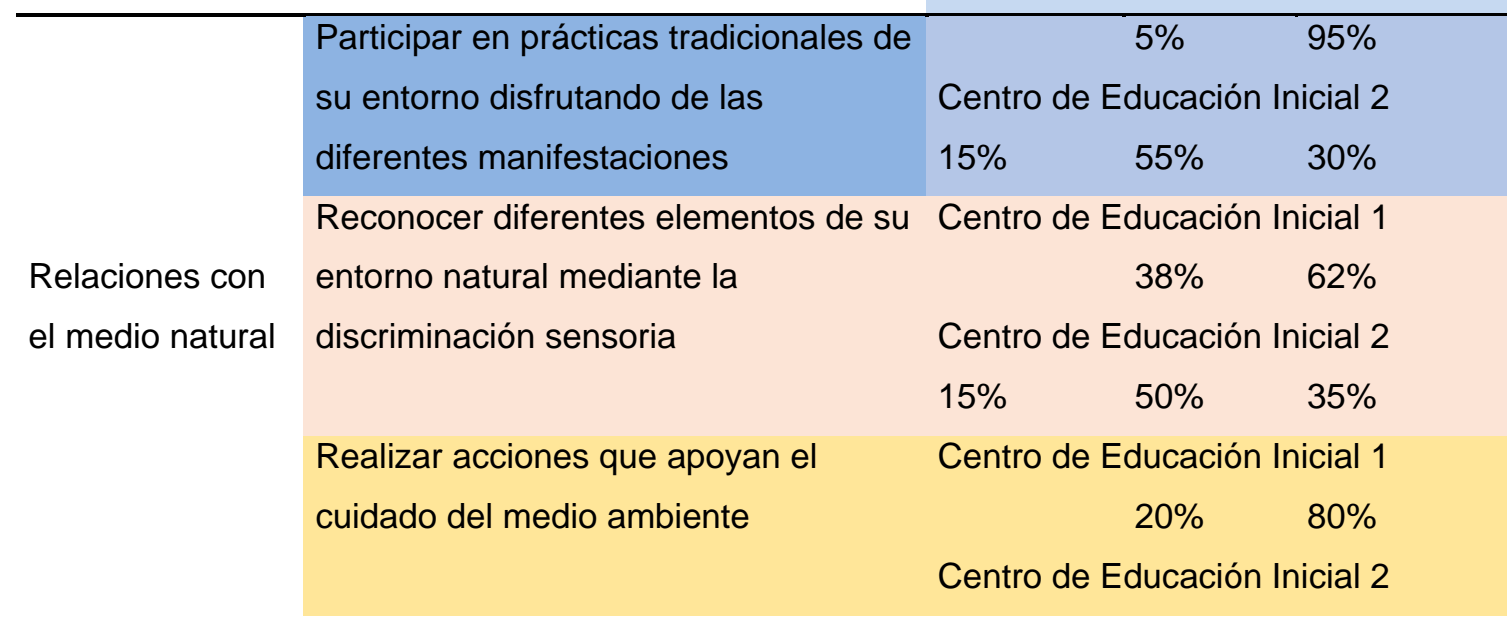




$$
20 \% \quad 80 \%
$$

Comprensión y

expresión del

lenguaje
Responder preguntas sobre un texto

narrado por el adulto, basándose en

los textos que observa.
Centro de Educación Inicial 1

$15 \%$ $85 \%$

Centro de Educación Inicial 2

$15 \%$
$65 \%$
$20 \%$

En la etapa del pretest se tomaron en cuenta las destrezas que coincidieron en la evaluación de los ámbitos y como se observa en los resultados, tanto en el ámbito de relaciones con el medio natural y cultural como en el ámbito de comprensión y expresión del lenguaje en los dos centros, existen un porcentaje de niños cuyas destrezas estaban en inicio ó en proceso, aquí se tomó en cuenta que la evaluación fue un mes antes de terminar el año escolar, por lo que se requirió de una intervención educativa.

\section{2da Etapa: Intervención}

En esta etapa de intervención educativa se realizaron un conjunto de planificaciones curriculares, ejecutadas por los estudiantes de la licenciatura en Educación Inicial en su práctica preprofesional, las cuales se adaptaron a las necesidades e intereses de los niños atendiendo a las dificultades encontradas en el diagnóstico inicial o pretest, donde las planificaciones curriculares estuvieron dirigidas a desarrollar destrezas en los ámbitos de:

\section{Ámbito: Relación con el medio natural y cultural}

Las planificaciones incluyeron actividades para desarrollar las siguientes destrezas:

Participar en prácticas tradicionales de su entorno disfrutando de las diferentes manifestaciones: para trabajar esta destreza se utilizaron las TIC como herramienta fundamental, donde se proyectaron videos en relación a la actividad que se iba a ejecutar y luego se realizaron una serie de preguntas para saber si el niño entendió o no el tema. Además, se trabajó con material concreto realizando casas abiertas que facilitaron a los niños lograr el aprendizaje significativo, se utilizó también el juego simbólico permitiendo que los niños adquieran dicha destreza.

Reconocer diferentes elementos de su entorno natural mediante la discriminación sensorial: se utilizó mucho la salida pedagógica que permitió que el niño utilice todos sus sentidos y adquiera un aprendizaje significativo.

Realizar acciones que apoyan el cuidado del medio ambiente: para esta destreza se utilizó la salida pedagógica, que permitió al niño entender la importancia del cuidado del planeta y no solo eso sino también desarrollar el hábito de cuidar el medio ambiente y ayudar en la limpieza, también se hizo uso de las técnicas grafo-plásticas ya que son estrategias que permiten al niño adquirir la coordinación viso motriz y plasmar los conocimientos adquiridos. 


\section{Ámbito: Comprensión y expresión del lenguaje}

Responder preguntas sobre un texto narrado por el adulto, basándose en los para-textos que observa: para esta destreza se trabajó mucho en el rincón de lectura con pictogramas grandes y coloridos, que sean llamativos para el niño y que permita lograr el aprendizaje del tema.

Además, se propusieron estrategias para trabajar con los niños que presentaban las siguientes NEE:

Trastornos de comportamiento: brindarles apoyo en cada actividad realizada, ubicarles cerca de sus compañeros con mejores comportamientos, darles confianza y seguridad y a su vez motivarlos promoviendo el diálogo, además aplicar actividades motivadoras para que el niño se mantenga controlado y concentrado

Trastorno de déficit de atención: evitar llamarle la atención en público, iniciar la clase con actividades que favorezcan la atención, hacer contacto visual con el estudiante antes de llamarlo o de darle instrucciones; esto ayuda al autocontrol.

Dificultad motriz: para esta necesidad se elaboró un "colchón de habilidades" que tiene diferentes actividades como poner botones, cerrar cierres, además se trabajó mucho lo que es la técnica dáctilo-pintura que permite que el niño progrese en la motricidad fina.

Discapacidad Visual: Sentar al niño en la parte delantera, frente a la docente, sustituir la percepción visual de los objetos mediante el tacto y el oído, utilizar material concreto.

\section{3ra Etapa de valoración de resultados}

En la tabla 3 y 4 se presentan los resultados comparativos del pretest y postest, a partir de la ficha de observación aplicada a los niños.

\begin{tabular}{|c|c|c|c|c|c|c|c|}
\hline \multirow{3}{*}{ Ámbito } & \multirow{3}{*}{ Destrezas } & \multirow{2}{*}{\multicolumn{3}{|c|}{$\begin{array}{l}\text { Resultados del } \\
\text { Pretest }\end{array}$}} & \multirow{2}{*}{\multicolumn{3}{|c|}{$\begin{array}{l}\text { Resultados del } \\
\text { Postest }\end{array}$}} \\
\hline & & & & & & & \\
\hline & & 1 & $P$ & $A$ & I & $\mathrm{P}$ & $A$ \\
\hline \multirow{8}{*}{$\begin{array}{l}\text { Relación con el } \\
\text { medio natural }\end{array}$} & Participar en prácticas & \multicolumn{6}{|c|}{ Centro de Educación Inicial 1} \\
\hline & tradicionales de su entorno & & $5 \%$ & $95 \%$ & & & $100 \%$ \\
\hline & disfrutando de las diferentes & \multicolumn{6}{|c|}{ Centro de Educación Inicial 2} \\
\hline & manifestaciones & $15 \%$ & $55 \%$ & & & $15 \%$ & $85 \%$ \\
\hline & Reconocer diferentes & \multicolumn{6}{|c|}{ Centro de Educación Inicial 1} \\
\hline & elementos de su entorno & & $38 \%$ & $62 \%$ & & $10 \%$ & $90 \%$ \\
\hline & natural mediante la & \multicolumn{6}{|c|}{ Centro de Educación Inicial 2} \\
\hline & discriminación sensoria & $15 \%$ & $50 \%$ & $35 \%$ & & $10 \%$ & $90 \%$ \\
\hline
\end{tabular}




$\begin{array}{lccc} & \text { Centro de Educación Inicial } 1 \\ \text { Realizar acciones que apoyan } & 20 \% \quad 80 \% & 10 \% & 90 \% \\ \text { el cuidado del medio ambiente } & \text { Centro de Educación Inicial } 2 & \\ & 20 \% \quad 80 \% & 40 \% & 60 \%\end{array}$

Tabla 3:

Resultados comparativos del Pretest y el postest para analizar el desarrollo de destrezas en los niños, en el ámbito Relación con el medio natural y cultural

Tabla 4:

Resultados comparativos del Pretest y el postest para analizar el desarrollo de destrezas en los niños, en el ámbito Comprensión y Expresión del Lenguaje

\begin{tabular}{|c|c|c|c|c|c|c|c|}
\hline \multirow{3}{*}{ Ámbito } & \multirow{3}{*}{ Destreza } & \multicolumn{6}{|c|}{ Resultados del } \\
\hline & & \multicolumn{3}{|c|}{ Pretest } & \multicolumn{3}{|c|}{ Resultados del Postest } \\
\hline & & 1 & $\mathrm{P}$ & A & I & $P$ & $A$ \\
\hline \multirow{4}{*}{$\begin{array}{l}\text { Comprensión y } \\
\text { expresión del } \\
\text { lenguaje }\end{array}$} & \multirow{4}{*}{$\begin{array}{l}\text { Responder preguntas } \\
\text { sobre un texto narrado por } \\
\text { el adulto, basándose en los } \\
\text { para textos que observa. }\end{array}$} & \multicolumn{6}{|c|}{ Centro de Educación Inicial 1} \\
\hline & & & $15 \%$ & $85 \%$ & & $5 \%$ & $95 \%$ \\
\hline & & \multicolumn{6}{|c|}{ Centro de Educación Inicial 2} \\
\hline & & $15 \%$ & $65 \%$ & $20 \%$ & & $5 \%$ & $95 \%$ \\
\hline
\end{tabular}

En la parte izquierda de las tablas 3 y 4 se presentan los resultados iniciales con respecto al logro de las destrezas de los niños, mientras que, en la parte derecha, se muestran los resultados de la aplicación de la medición postest. Después de la intervención se pudo evidenciar a través de los resultados obtenidos que hubo una mejora en cuanto a las siguientes destrezas; "participar en prácticas tradicionales de su entorno disfrutando de las diferentes manifestaciones" donde en el CEI 1 en el pretest existía un $5 \%$ en proceso y un $95 \%$ en alcanzado, pero en el postest aumento a un $100 \%$ en alcanzado, de igual manera en el CEI 2 existía un 15\% en inicio, un 50\% en proceso y solo un $35 \%$ en alcanzado, para el postest se observa que se disminuye a un $0 \%$ en iniciado, así como $15 \%$ en proceso y se aumenta a un $85 \%$ en alcanzado dando como resultado que la mayoría de los infantes logren alcanzar un nivel de adquirido, en las destrezas observadas, todo esto a través de las diferentes planificaciones y estrategias pedagógicas que fueron aplicadas permitiendo atender a cada niño tomando en cuenta el ritmo y estilo de aprendizaje.

\section{CONCLUSIONES}

Se evidencia con los resultados comparativos de la evaluación entre el pretest y el postest que existe una mejora significativa en el desarrollo de las destrezas de los ámbitos de desarrollo y aprendizaje que fueron analizados en los niños de la muestra estudiada, lo que evidencia que la evaluación fue fundamental en todo el proceso educativo ya que, a través de 
los instrumentos, se pudo detectar tanto los logros como las NEE de cada niño o los signos de alerta que indicaron alguna dificultad y mediante el diagnóstico se buscaron actividades y estrategias que a través de la intervención permitieron lograr el aprendizaje significativo. Estos resultados corroboran lo mencionado por López (2015) sobre la evaluación quien señaló que:

Todos los niveles de educación tienen características que lo diferencian del resto, por tanto, en educación inicial se asume una evaluación que corresponde con las características que manifiestan los niños y niñas de cero a seis años de edad. Es bien sabido que la evaluación es un proceso sistemático que se lleva a cabo de manera paulatina y constante en diversos momentos de la rutina diaria, a lo largo del año escolar, siendo en primer momento propicia para diagnosticar las necesidades, intereses, fortalezas, nivel de desarrollo real y de desarrollo próximo de los y las estudiantes, la noción de todo esto, ayuda a las docentes a elegir los componentes, objetivos, aprendizajes a ser alcanzados y las estrategias a desarrollar para favorecer el desarrollo integral de los niños y niñas (p. 47).

De los análisis realizados podemos concluir que:

En los centros de educación inicial estudiados los procesos de evaluación que se aplican por los docentes, no siempre consideran las necesidades educativas especiales y el ritmo y estilo de los estudiantes.

Es imprescindible conocer los instrumentos para la evaluación en Educación Inicial, así como la manera de aplicarlos de forma continua para detectar logros y necesidades en los niños que permitan tomar decisiones y favorecer la mejora de las insuficiencias que presenten en el desarrollo de sus destrezas.

Es importante la capacitación a los docentes sobre cómo abordar e intervenir con los niños que presentan NEE con el fin de lograr el aprendizaje integral de cada educando.

La preparación de los docentes sobre las estrategias pedagógicas, que pueden utilizarse atendiendo a los diferentes tipos de NEE, es muy necesaria para que posean las herramientas que le permitan adaptar el proceso de enseñanza aprendizaje a las necesidades de cada infante.

\section{REFERENCIAS BIBLIOGRAFICAS}

Altamirano, J. (2015). Evaluación de los Aprendizajes Infantiles.

http://www.runayupay.org/publicaciones/evaluacion_de_los_aprendizajes_infantiles.pdf

Bonilla, R. E. (2015). Capacitación a educadoras para promover la inclusión de los niños con necesidades educativas especiales en los Centros de Educación Inicial de la parroquia Simón Plata Torres de la Ciudad de Esmeraldas [Pontificia Universidad Católica del Ecuador, Sede Esmeraldas]. https://repositorio.pucese.edu.ec/bitstream/123456789/537/1/BONILLA GONZALEZ ROSARIO ELVIRA.pdf 
Cabrera Jara Hilda Valeria. (2015). Procesos evaluativos utilizados por los docente para los niños y niñas de 4 a 5 años en los Centros de Desarrollo Inicial privados del cantón Cuenca [Univeridad del Azuay]. http://dspace.uazuay.edu.ec/handle/datos/7507

Cisneros, E. (2017). Programa de inclusion educativa para la atencion de Necesidaades educativas especiales [Pontificia Universidad Católica del Ecuador]. http://repositorio.puce.edu.ec/bitstream/handle/22000/13997/tesis ELEANA CISNEROS.pdf?sequence $=1$ \&isAllowed $=y$

Lopez, I., \& Venezuela, G. (2015). Niños y adolecentes con necesidades educativas especiales. Revista Medica Clinica Condes, 2(1), 42-51. https://www.elsevier.es/esrevista-revista-medica-clinica-las-condes-202-pdf-S0716864015000085

López, R. (2015). Planificación y evaluación en el nivel de educación Inicial [Universidad de Carabobo]. http://hdl.handle.net/123456789/948

Luque, D. (2009). Las necesidades educativas especiales como necesidades básicas. Revista Latinoamericana de Estudios Educativos, 39(3), 201-213. http://www.redalyc.org/articulo.oa?id=27015078009

Ministerio de Educación de Ecuador. (2011). Estrategias pedagógicas para atender a las necesidades educativas especiales en la educación regular. http://www.colegiorohde.edu.ec/descargas/TALLERES-2016/Inclusi\%F3n adaptaci\%F3n/NEE. ESTRATEGIAS PEDAG_GICAS.pdf

Ministerio de Educación del Perú. (2006). Guía de evaluación de educación inicial. https://www.oei.es/historico/inicialbbva/db/.../guia_evaluacion_educacion_inicial.pdf

Ministerio de Educación Ecuador (MinEduc). (2013). Adaptaciones curriculares para estudiante con Necesidades Educativa especiales.

Ministerio de Educación Ecuador (MinEduc). (2014). Curriculo de educación inicial. https://educacion.gob.ec/wp-content/uploads/downloads/2016/03/CURRICULO-DEEDUCACION-INICIAL.pdf

Morre, D. (2006). Transtorno de la conducta una guia de intervencion en la escuela. http://www.psie.cop.es/uploads/aragon/Aragón-trastornos-de-conducta-una-guia-deintervencion-en-la-escuela.pdf

Secretaría de Estado de Educación de República Dominicana. (2006). La Evaluación Educativa en el Nivel Inicial. http://sitios.educando.edu.do/biblioteca/components/com_booklibrary/ebooks/La_Evalu acion_Educativa_NI.pdf

Villacis, J. (2018). Derecho a la educación inclusiva de los niños, niñas y adolescentes con necesidades educativas especiales no asociadas a la discapacidad, en la legislacion educativa [Universidad Central del Ecuador]. http://www.dspace.uce.edu.ec/handle/25000/15244 